\title{
Universal Relaxation Governs the Nonequilibrium Elasticity of Biomolecules
}

\author{
Christian Kappel, ${ }^{1}$ Nicole Dölker, ${ }^{1,2}$ Rajendra Kumar, ${ }^{1}$ Mareike Zink, ${ }^{1,3}$ Ulrich Zachariae,,${ }^{1,4}$ and Helmut Grubmüller ${ }^{1, *}$ \\ ${ }^{1}$ Theoretical \& Computational Biophysics, MPI for Biophysical Chemistry, Am Fassberg 11, 37077 Göttingen, Germany \\ ${ }^{2}$ Computational Biophysics Group, CNIO, 28029 Madrid, Spain \\ ${ }^{3}$ Department of Physics and Earth Sciences, University of Leipzig, 04103 Leipzig, Germany \\ ${ }^{4}$ SUPA, School of Physics and Astronomy, University of Edinburgh, Edinburgh EH9 3JZ, United Kingdom \\ (Received 13 May 2011; revised manuscript received 26 February 2012; published 14 September 2012)
}

Experimental and computational dynamic force spectroscopy is widely used to determine the mechanical properties of single biomolecules. Whereas so far the focus has mainly been on rupture or unfolding forces, recent force-probe molecular dynamics simulations have revealed a strong loading rate dependence of biomolecular elasticities, which cannot be explained by the established one-dimensional transition-state treatments. We show that this nonequilibrium behavior can be explained by a theory that includes relaxation effects. For three structurally and mechanically quite diverse systems, a single relaxation mode suffices to quantitatively describe their loading-rate-dependent elastic behavior. Atomistic simulations of these systems revealed the microscopic nature of the respective relaxation modes. This result suggests a new type of "elasticity spectroscopy" experiment, which should render nonequilibrium properties of structured macromolecules accessible to single-molecule force spectroscopy.

DOI: 10.1103/PhysRevLett.109.118304

Molecular-scale forces govern fundamental biomolecular processes such as ligand binding, the motion of molecular motors, and protein folding [1-3]. The mechanical response of macromolecules to small external forces can be probed by dynamic force spectroscopy and optical tweezers. For a wide range of macromolecules, these single-molecule experiments have impressively confirmed time-dependent transition-state theories predicting a logarithmic increase of rupture or unfolding forces with the loading rate [4-8].

First established by Bell [9], most of these theories rely on a one-dimensional transition state or Kramers treatment for activated barrier crossings [10], which assumes all molecular degrees of freedom except the probe coordinate to be in equilibrium, even at the barrier top $[5-8,11,12]$. As an important consequence, current models predict a Hookean behavior of structured biomolecules, i.e., an elastic response independent of the loading rate. This assumption, however, is difficult to reconcile with the large number of known slow internal dynamic modes particularly of biomolecules.

For protein folding, recent single-molecule spectroscopy experiments and their theoretical treatments demonstrated the need to go beyond approaches with one-dimensional reaction coordinates [13]. In particular, both the use of multiple pathways $[13,14]$ in the treatment of protein folding and two-dimensional transition-state treatments of yielding under constant load [15-17] have shown strong deviations from one-dimensional results. Elastic properties of biomolecules, however, have not yet been considered.

With recent force-probe molecular dynamics simulations focusing on the time-dependent elastic properties of biomolecules rather than their yielding behavior, this discrepancy has become acute. Indeed, strong and so far
PACS numbers: 82.37.Rs, 82.37.Gk, 87.10.Pq, 87.10.Tf

unexplained non-Hookean deviations from Bell-type models have been observed for several proteins with quite diverse structural and elastic properties, e.g., a flexible superhelical protein [18,19], a rigid virus capsid [20,21], and the bacteriophage head-tail connector $\Phi 29$ [22]. To address this issue, and going beyond transition-state theory, we here develop a nonequilibrium theory that includes relaxation of modes perpendicular to the reaction coordinate. A single relaxation mode suffices to quantitatively describe the loading-rate-dependent elastic properties of the above prototypic structured macromolecules, suggesting that this property may be universal.

This result also suggests a new experimental approach. Whereas so far almost all single-molecule force-probe experiments have focused on unbinding, rupture, or unfolding forces, nonequilibrium relaxation dynamics and molecular friction in biological macromolecules should become accessible through "elasticity spectroscopy," i.e., by measurement of their loading-rate-dependent elastic properties.

According to Bell's theory [9], deformation and rupture occur on a single reaction coordinate $x$, governed by a Gibbs free-energy landscape $G_{0}(x)$ [Fig. 1(a)]. The region near the minimum of $G_{0}(x)$ at $x=0$ is the equilibrium reactant state of the system, and yielding occurs between that minimum and a barrier located at $x_{b}$, the rupture length. With an attempt frequency $\omega_{0}$, a logarithmic, force-dependent rupture rate $r(F)=\omega_{0} \exp \left(\beta F x_{b}\right)$ is predicted.

The external force exerted onto the system in force-probe experiments such as single-molecule atomic force spectroscopy or optical tweezers is included via a harmonic "spring" coupling described by a time-dependent probe potential, $V_{p}=\frac{1}{2} k_{p}(x-v t)^{2}$. Assuming a constant loading rate, the 

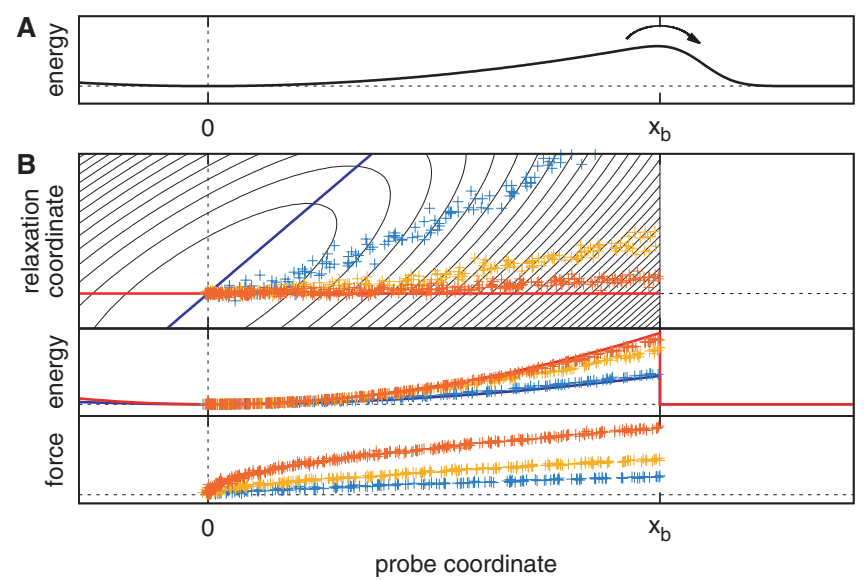

FIG. 1 (color). Models for rupture events. (a) One-dimensional Bell-type model. (b) Two-dimensional nonequilibrium model. Upper panel: Free-energy landscape $G_{0}(x, y)$ (contour lines), Eq. (2), with a barrier at $x=x_{b}$ and sample paths (symbols). Probe potentials $V_{p}=\frac{1}{2} k_{p}(x-v t)^{2}$ with slow (blue, top path), medium (orange, mid), and fast (red, bottom) retraction velocities were applied. Limiting cases for fast and slow pulling are depicted as red (horizontal) and blue (diagonal) lines, respectively. Center panel: Potential energy along the sample paths (symbols, top: fast, bottom: slow) and limiting cases. Lower panel: Forces along the sample paths.

minimum of $V_{p}$ moves with constant velocity $v$. Neglecting barrier recrossings as well as the small perturbation of the probability distribution caused by "leakage" over the barrier, the probability distribution of the reaction coordinate within the reactant state, $p(x, t)$, obeys a Smoluchowski equation for the time-dependent free energy $G(x, t)=G_{0}(x)+V_{p}(x, t)$. With the use of an appropriate barrier crossing rate $r(t)$, an expression for the probability of finding the system within the reactant state $P(t)$ is readily derived. This results in a distribution of yielding forces and, in particular, an average yielding force $\left\langle F_{\text {yield }}(v)\right\rangle \sim \ln \left(v / v_{0}\right)$ scaling logarithmically with probe velocity $v$ (with appropriate unit velocity $v_{0}$ ) [4,6-9].

Similarly, for the time span prior to yielding, the onedimensional, near-equilibrium treatment predicts an increasing average force

$\left\langle F_{v}(t)\right\rangle=k_{p} v\left[t-\frac{k_{p}}{k_{0}+k_{p}}\left(t-\frac{1-e^{-\beta\left(k_{0}+k_{p}\right) D t}}{\beta\left(k_{0}+k_{p}\right) D}\right)\right]$,

which enables extraction of the elastic constant $k_{0}=$ $\left.\partial_{x}^{2} G_{0}(x)\right|_{x=0}$ from force-extension data from experiments or force-probe simulations. Obviously, this one-dimensional model predicts an elastic constant that does not vary with loading rate, in contrast to the velocitydependent behavior we observed [21].

To resolve this discrepancy, we include slow nonequilibrium relaxation dynamics of modes perpendicular to the probe coordinate (slow on the time scale of the pulling process). Accordingly, a generalized model is considered [Fig. 1(b), contour lines],

$$
G_{0}(x, y)=\frac{1}{2} k_{\|} x^{2}-k_{\perp} \alpha x y+\frac{1}{2} k_{\perp} \alpha^{2} y^{2},
$$

for $x<x_{b}$ and 0 otherwise. Here, $x$ is the probe coordinate and $y$ represents one or several, typically collective, relaxation coordinates perpendicular to $x . k_{\|}$and $k_{\perp}$ are the corresponding force constants with coupling constant $\alpha$. To enable analytic treatment, a coupled harmonic model is used.

In this generalized treatment, the path taken by the system on the time-dependent free-energy surface, governed by the probe potential $V_{p}$ moving along the probe coordinate $x$, depends on the probe velocity $v$ [Fig. 1(b), colored symbols]. At low probe velocities, all orthogonal modes $y$ remain close to their equilibrium distribution, and the system follows a minimum energy path (blue symbols, top paths). By construction, this generalized model reduces to the one-dimensional Bell-type model

$$
G_{0, \text { Bell }}(x)=\frac{1}{2}\left(k_{\|}-k_{\perp}\right) x^{2},
$$

in the quasistatic limit of fully equilibrated orthogonal coordinates $y$ (diagonal blue line). For larger $v$ values, the slow modes can no longer equilibrate, and thus the path taken deviates from the minimum energy pathway (orange, mid paths). In the adiabatic limit of infinitely slow relaxation, the path approaches a parallel to the probe coordinate (red, bottom paths). As a result, an increased barrier height and stronger forces are found for deformation and yielding of the system.

By including the time-dependent probe potential, $V_{p}$, $G(\mathbf{x}, t)=G_{0}(\mathbf{x})+V_{p}(x, t)$ can be cast into the more convenient matrix form

$$
G(\mathbf{x}, t)=\frac{1}{2}(\mathbf{x}-\mathbf{v} t)^{T} \mathbf{C}(\mathbf{x}-\mathbf{v} t)+V_{0}(t),
$$

with $C_{11}=k_{\|}+k_{p}, C_{12}=C_{21}=-\alpha k_{\perp}, C_{22}=\alpha^{2} k_{\perp}$, $\mathbf{x}=(x, y)^{T}$, and $\mathbf{v}=v(1,1 / \alpha)^{T} /\left[1+\left(k_{\|}-k_{\perp}\right) / k_{p}\right] . V_{0}(t)$ is an extraneous time-dependent offset. The two-dimensional probability distribution $p(\mathbf{x}, t)$ obeys a Smoluchowski equation

$$
\partial_{t} p(\mathbf{x}, t)=\nabla D e^{-\beta G(\mathbf{x}, t)} \nabla e^{\beta G(\mathbf{x}, t)} p(\mathbf{x}, t),
$$

with diffusion constant $D$ and the reciprocal thermal energy $\beta$, which is readily solved assuming an underlying OrnsteinUhlenbeck process $p_{\mathrm{OU}}(\mathbf{x}, t)$ [23], after transformation into a Smoluchowski equation with time-independent potential via

$$
p(\mathbf{x}, t)=p_{\mathrm{OU}}\left(\mathbf{x}+\mathbf{x}_{\text {off }}-\mathbf{v} t, t\right) .
$$

Here, $\mathbf{x}_{\text {off }}=\sum_{i=1,2}\left(\mathbf{e}_{i} \cdot \mathbf{v}\right) \mathbf{e}_{i} /\left(\beta \lambda_{i} D\right)$, where $\lambda_{1,2}$ are the eigenvalues and $\mathbf{e}_{1,2}$ are the normalized eigenvectors of $\mathbf{C}$. After subsequent diagonalization, Eq. (6) reduces to two uncoupled one-dimensional Ornstein-Uhlenbeck processes, with an analytic solution $p(\mathbf{x}, t)$ in terms of Gaussian functions with time-dependent widths and centers. The latter are given by 


$$
\langle\mathbf{x}(t)\rangle=\sum_{i=1,2}\left(\mathbf{e}_{i} \cdot \mathbf{v}\right) \mathbf{e}_{i}\left(t-\frac{1-e^{-\beta \lambda_{i} D t}}{\beta \lambda_{i} D}\right) .
$$

From the $x$ component, a mean force along the probe coordinate

$$
\langle F(t)\rangle=k_{p}(v t-\langle x(t)\rangle),
$$

is obtained. With the analytic expression for $p(\mathbf{x}, t)$ and the approximate instantaneous transition rate $[10,24]$

$$
r(t)=\frac{D}{\int_{\text {well }} e^{-\beta G_{\mathrm{NE}}(\mathbf{x}, t)} d x \int_{\text {barrier }} e^{\beta G_{\mathrm{NE}}(\mathbf{x}, t)} d x},
$$

where $G_{\mathrm{NE}}(\mathbf{x}, t)=-k_{B} T \ln p(\mathbf{x}, t)$ is (up to an irrelevant offset) the nonequilibrium free energy, one obtains (see Supplemental Material [25])

$$
r(t)=\frac{2 D \tau e^{-\tau^{2}}}{\sqrt{\pi} \sigma_{x}^{2}(t)(1+\operatorname{erf}(\tau))\left(1+e^{-2 \tau^{2}}\right)},
$$

where $\tau=\left(x_{b}-\langle x(t)\rangle\right) /\left(\sqrt{2} \sigma_{x}(t)\right) ; \sigma_{x}(t)$ is defined in the Supplemental Material [25].

The probability $P(t)$ of finding the system within the reactant well at time $t$ is obtained from numerical integration of

$$
\partial_{t} P(t)=-r(t) P(t)
$$

which yields an average velocity-dependent yielding force

$$
\left\langle F_{\text {yield }}(v)\right\rangle=-\int_{0}^{\infty}\langle F(t)\rangle \partial_{t} P(t) d t .
$$

In agreement with Bell's model and its extensions, the rate dependence of $\left\langle F_{\text {yield }}(v)\right\rangle$ is governed by logarithmic terms in $v$. Here however, $\langle F(t)\rangle$ exhibits also loading-ratedependent elastic properties.

We applied our model to the $\operatorname{Importin}-\beta(\operatorname{Imp} \beta)$ superhelix, a protein characterized by its unusual intrinsic flexibility $[19,26]$. By force-probe all-atom molecular dynamics simulations, $\operatorname{Imp} \beta$ was reversibly stretched by up to twice its equilibrium length with probe velocities from 0.08 to $20 \mathrm{~m} / \mathrm{s}$ [19]. At low probe velocities, effective elastic constants $k_{\text {eff }}$ obtained from force-extension curves converged to $(10 \pm 4) \times 10^{-3} \mathrm{~N} / \mathrm{m}$, in agreement with the value derived from the end-to-end distance distribution of $\operatorname{Imp} \beta$ in equilibrium simulations. At high probe velocities, however, a clear velocity dependence is seen [Fig. 2(a)], in obvious contradiction to Bell's model.

We thus investigated whether the behavior of the system across all probe velocities can be explained by our relaxation model. Accordingly, Eq. (8) was simultaneously fit to all 42 force profiles obtained, yielding $k_{\|}=$ $0.101 \mathrm{~N} / \mathrm{m}, k_{\perp}=0.090 \mathrm{~N} / \mathrm{m}, \alpha=0.510$, and $D=1.641 \times$ $10^{-10} \mathrm{~m}^{2} / \mathrm{s}$ [Fig. 2(b), solid lines]. The resulting equilibrium elastic constant $k_{\mathrm{eq}}=k_{\|}-k_{\perp}=11 \times 10^{-3} \mathrm{~N} / \mathrm{m}$ agrees well with the equilibrium elastic constant obtained previously from a one-dimensional model, $(10 \pm 4) \times 10^{-3} \mathrm{~N} / \mathrm{m}$.

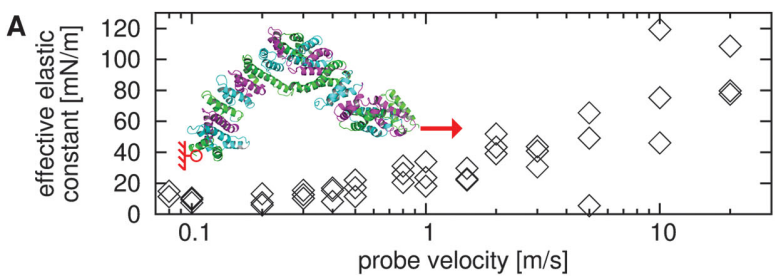

B

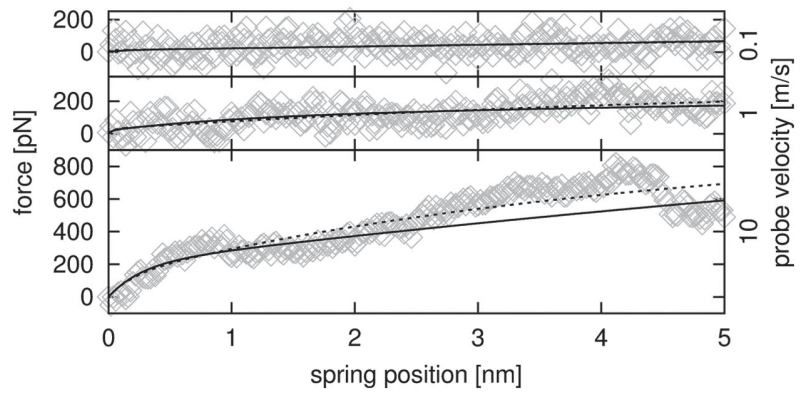

FIG. 2 (color). (a) Velocity-dependent effective elastic constants $k_{\text {eff }}$ derived from $\operatorname{Imp} \beta$ force-probe MD simulations. Elastic constants were calculated with the assumption of an underlying one-dimensional energy landscape $G_{0}(x)=\frac{1}{2} k_{\text {eff }} x^{2}$ using Eq. (1) (b) Example force profiles from stretching MD simulations (symbols) with fits using Eq. (8) assuming a two-dimensional energy landscape $G_{0}(x, y)$ according to Eq. (2) (lines). Solid lines show a collective fit to all available force profiles, dashed lines display individual fits to each.

The diffusion constant yields a molecular friction coefficient of $k_{B} T / D=2.5 \times 10^{-11} \mathrm{~N} \mathrm{~s} / \mathrm{m}$ for the respective mode. For comparison, and to obtain optimal reference parameters, Eq. (8) was fit to each force curve individually [Fig. 2(b), dashed lines]. These fits reproduce the curves from the simulations remarkably well. Notably, force curves from the collective fit (solid lines) closely resemble these reference curves. Hence, using our model, four parameters suffice to describe the nonequilibrium elastic response of the protein to an external force over a broad range of probe velocities. Closer analysis of the atomistic force-probe simulations revealed as a perpendicular relaxation mode collective tilting motions of adjacent $\alpha$-helical units [colored in Fig. 2(a)].

We next addressed the local elasticity of a virus capsid, which is much stiffer than $\operatorname{Imp} \beta$. The Southern Bean Mosaic Virus (SBMV) was chosen, as its elastic properties were recently characterized by all-atom force-probe molecular dynamics simulations with probe velocities ranging from 2 to $50 \mathrm{~m} / \mathrm{s}$ [21,27]. Figure 3(a) shows the effective elastic constants obtained from linear fits to the force-time curves. Although this macromolecular system is very different from $\operatorname{Imp} \beta$, all effective elastic constants derived from the simulations are reproduced [Fig. 3(a), black line] and yield an equilibrium stiffness of $k_{\text {eq }}=k_{\|}-k_{\perp}=$ $0.954 \mathrm{~N} / \mathrm{m}$.

To test the model's predictions, we used the parameters $k_{\|}, k_{\perp}, \alpha$, and $D$ obtained from the previous fit to calculate the velocity dependence of yielding forces of the SBMV 


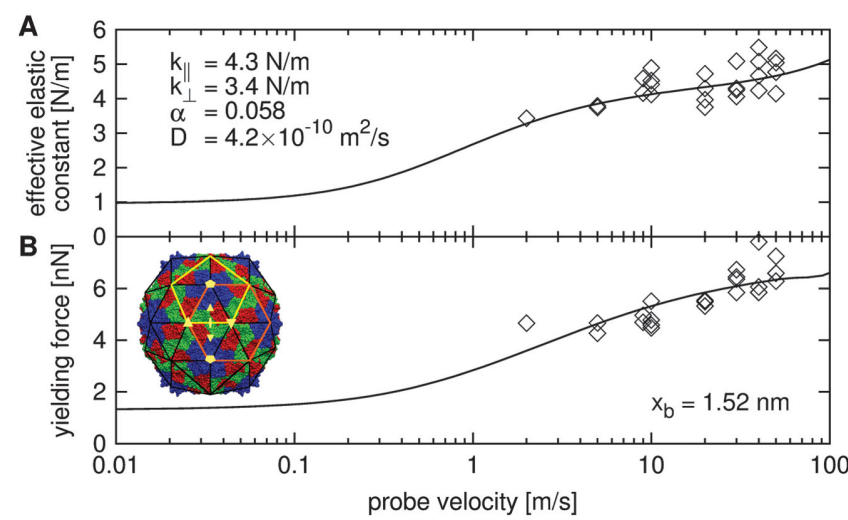

FIG. 3 (color). Effective elastic constants and yielding forces from SBMV force-probe MD simulations. (a) Velocitydependent elastic constants $k_{\text {eff }}$ (symbols) with a fit of Eq. (8) (line). (b) Yielding forces with a fit to Eq. (12).

shell [Fig. 3(b)]. With the barrier location $x_{b}$ as sole additional fit parameter, the logarithmic dependence of yielding forces was reproduced for all investigated velocities. As expected for velocities below the characteristic relaxation time, a near-constant yielding force of $\sim 1.4 \mathrm{nN}$, which lies in the range measured for viral capsids $(\sim 0.6-2.5 \mathrm{nN})$, is predicted [20,28].

To test the predictive value of the relaxation model, we analyzed the elastic behavior of the $\Phi 29$ head-tail connector [22] (Fig. 4), for which velocity-dependent elastic constants are also observed for the distance $L$ between the upper and lower domains as force-probe coordinate. Here, an irislike twisting motion provides a likely relaxation mode, which followed a velocity-dependent path [Fig. 4(a) symbols]. Analysis of this path enabled us to extract the parameters for our model without using any force or extension curves as input. To that aim, the probability distribution $p(L, \theta)$ from equilibrium simulations was fit to $\exp \left[-\beta G_{0}(L, \gamma \theta)\right]$, yielding $k_{\|}=24.3 \mathrm{~N} / \mathrm{m}, \quad k_{\perp}=2.1 \mathrm{~N} / \mathrm{m}, \quad$ and $\gamma \alpha=$ -0.15 , where $\gamma$ is a conversion factor [Fig. 4(a), contour lines]. With these parameters, a simultaneous fit to all trajectories was carried out, yielding $\gamma=0.13 \mathrm{~nm} / \mathrm{deg}$ and $D=1.09 \times 10^{-12} \mathrm{~m}^{2} / \mathrm{s}$ [Fig. 4(a), lines]. As can be seen in the Figure, the obtained model accurately describes the molecular dynamics trajectories. Moreover, also the predicted forces agree well with those from the simulations [Fig. 4(b)], thus confirming the validity and predictive power of our model.

We have shown that universal nonequilibrium relaxation of one collective mode quantitatively explains the inelastic and elastic force response of quite diverse structured biological macromolecules. Combined with atomistic simulations, the microscopic origin of the proposed relaxation coordinate is revealed as collective modes, thus connecting atomistic descriptions to more phenomenological descriptions of nonclassical mechanical behavior, as used, e.g., in material science [29]. Thereby, our study extends previous work on

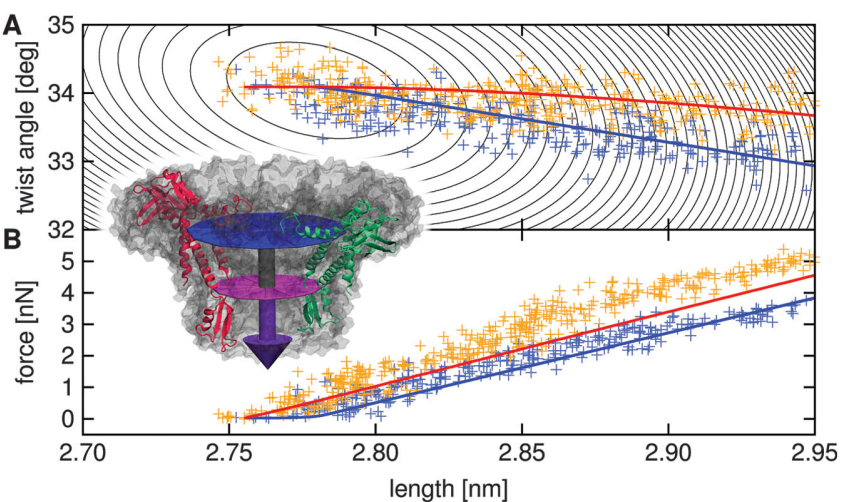

FIG. 4 (color). Force-probe simulations of $\Phi 29$ head-tail connector. (a) Velocity-dependent paths (symbols) observed during fast (orange, top paths, $v=4 \mathrm{~m} / \mathrm{s}$ ) and slow (blue, bottom paths, $v=0.2 \mathrm{~m} / \mathrm{s}$ ) pulling simulations and fits to Eq. (7) (red and blue lines for slow and fast probe velocities, respectively). (b) Corresponding forces (symbols) and force predictions according to Eq. (8) based on a fit to the trajectories (lines).

biomolecular yielding beyond one-dimensional reaction coordinates [13-17] into a general two-dimensional treatment of molecular elasticity. Our theory is readily generalized to multiple dimensions and a broad spectrum of relaxation processes. The described non-Hookean fingerprint should be detectable in force-probe experiments on systems with millisecond or slower relaxations. By "elasticity spectroscopy," one should be able to extract, e.g., coupling strengths of perpendicular relaxation modes, as well as their relaxation rates and stiffness.

We acknowledge funding by HFSP (RGP 53/2004) and the EU (FP6-2004-NEST-PATH, Nanomot, and FP7 Grant Agreement No. 211800). We thank Wilson C. K. Poon for critically reading the manuscript.

*hgrubmu@gwdg.de

[1] V. Moy, E. Florin, and H. Gaub, Science 266, 257 (1994).

[2] J. Molloy, J. Burns, J. Kendrick-Jones, R. Tregear, and D. White, Nature (London) 378, 209 (1995).

[3] M. Kellermayer, S. Smith, H. Granzier, and C. Bustamante, Science 276, 1112 (1997).

[4] O. K. Dudko, G. Hummer, and A. Szabo, Phys. Rev. Lett. 96, 108101 (2006).

[5] G. Hummer and A. Szabo, Biophys. J. 85, 5 (2003).

[6] E. Evans and K. Ritchie, Biophys. J. 72, 1541 (1997).

[7] S. Izrailev, S. Stepaniants, M. Balsera, Y. Oono, and K. Schulten, Biophys. J. 72, 1568 (1997).

[8] B. Heymann and H. Grubmüller, Phys. Rev. Lett. 84, 6126 (2000).

[9] G. I. Bell, Science 200, 618 (1978).

[10] H. A. Kramers, Physica B+C (Amsterdam) 7, 284 (1940).

[11] H. Grubmüller, B. Heymann, and P. Tavan, Science 271, 997 (1996).

[12] U. Seifert, Phys. Rev. Lett. 84, 2750 (2000). 
[13] R. B. Best and G. Hummer, Proc. Natl. Acad. Sci. U.S.A. 107, 1088 (2010).

[14] K. Lindorff-Larsen, S. Piana, R. O. Dror, and D. E. Shaw, Science 334, 517 (2011).

[15] Y. Suzuki and O. K. Dudko, Phys. Rev. Lett. 104, 048101 (2010).

[16] Y. Suzuki and O. K. Dudko, J. Chem. Phys. 134, 065102 (2011).

[17] J. D. Chodera and V.S. Pande, Phys. Rev. Lett. 107, 098102 (2011).

[18] J. Forwood, A. Lange, U. Zachariae, M. Marfori, C. Preast, H. Grubmüller, M. Stewart, A. Corbett, and B. Kobe, Structure 18, 1171 (2010).

[19] C. Kappel, U. Zachariae, N. Dölker, and H. Grubmüller, Biophys. J. 99, 1596 (2010).

[20] W. H. Roos, R. Bruinsma, and G. J. L. Wuite, Nature Phys. 6, 733 (2010).
[21] M. Zink and H. Grubmüller, Biophys. J. 96, 1350 (2009).

[22] D. J. Müller, A. Engel, J. L. Carrascosa, and M. Velez, EMBO J. 16, 2547 (1997).

[23] G. E. Uhlenbeck and L. S. Ornstein, Phys. Rev. 36, 823 (1930).

[24] Z. Hu, L. Cheng, and B. J. Berne, J. Chem. Phys. 133, 034105 (2010).

[25] See Supplemental Material at http://link.aps.org/ supplemental/10.1103/PhysRevLett.109.118304 for a derivation of Eq. (10).

[26] U. Zachariae and H. Grubmüller, Structure 16, 906 (2008).

[27] M. Zink and H. Grubmüller, Biophys. J. 98, 687 (2010).

[28] W.H. Roos, I. L. Ivanovska, A. Evilevitch, and G. J. L. Wuite, Cell Mol. Life Sci. 64, 1484 (2007).

[29] W. L. Johnson and K. Samwer, Phys. Rev. Lett. 95, 195501 (2005). 